\title{
The Striped Phase in Presence of Disorder and Lattice Potentials
}

\author{
N. Hasselmann ${ }^{a, b}$, A. H. Castro Neto ${ }^{a}$, C. Morais Smith $^{b}$, and Y. Dimashko ${ }^{b}$ \\ ${ }^{a}$ Dept. of Physics, University of California, Riverside, CA, 92521, USA \\ ${ }^{b}$ I. Institut für Theoretische Physik, Universität Hamburg, D-20355 Hamburg, Germany
}

(July 14, 2017)

\begin{abstract}
We study the influence of disorder and lattice effects on the striped phase (SP) and the incommensurate (IC) spin fluctuations of the cuprates and nickelates. Starting from a phenomenological model of a discrete quantum string on a lattice with disorder, we derive the renormalisation group (RG) equations in leading order in the lattice and disorder strength. Three regimes are identified, the quantum membrane phase, the flat phase pinned by the lattice and the disorder pinned phase. We compare our results with measurements on nickelates and cuprates and find good agreement.
\end{abstract}

PACS numbers: 71.45.Lr, 74.20.Mn, 74.72.Dn, 75.30.F

The discovery of a static SP in the compound $\mathrm{La}_{1.6-\mathrm{x}} \mathrm{Nd}_{0.4} \mathrm{Sr}_{\mathrm{x}} \mathrm{CuO}_{4}$ at both non-superconducting $(x \simeq$ $1 / 8)$ [1] and superconducting $(x=0.15,0.20)$ [2] compositions has revived interest in the possibility that a similar, albeit fluctuating order may be common to all under- and optimally doped cuprate superconductors. The strongest indications for a fluctuating SP order exist for the $\mathrm{La}_{2-\mathrm{x}} \mathrm{Sr}_{\mathrm{x}} \mathrm{CuO}_{4}$ family, where the occurrence of incommensurate (IC) magnetic peaks in inelastic neutron scattering experiments has been observed for quite some time 3 [5]. Very recently, similar IC correlations were also measured in $\mathrm{YBa}_{2} \mathrm{Cu}_{3} \mathrm{O}_{7-\delta}$ [6]. Also, evidence for stripes in $\mathrm{Bi}_{2} \mathrm{Sr}_{2} \mathrm{CaCu}_{2} \mathrm{O}_{8+\delta}$ has been reported [7]. A static SP order has been detected in both $\mathrm{O}$ and $\mathrm{Sr}$ doped nickelates $\left(\mathrm{La}_{2-\mathrm{x}} \mathrm{Sr}_{\mathrm{x}} \mathrm{NiO}_{4+\delta}\right)$ [ 10 , which have a structure very similar to the cuprates. While the concept of a dynamical fluctuating SP accounts qualitatively for many properties of cuprate superconductors (e. g. the form of the Fermi surface 111, the IC spin fluctuations [12], or the suppression of the Néel temperature with doping [13]), it is at present theoretically not well understood. Even under the assumption that all excess holes are tightly bound in strings many questions regarding the nature of the fluctuations, the charge- and spin coherence of the ground state, and the influence of disorder or lattice potentials on the SP are still open.

In the long wavelength limit a sufficiently dilute array of classical stripes can be described as a membrane with anisotropic stiffnesses, where the stiffness in one direction is dominated by the entropic repulsion between neighboring stripes. In presence of disorder, such a membrane is pinned at all temperatures, leading to a glassy SP [14. Also, in the presence of a weak substrate lattice potential, a weakly IC phase near a $p \times 1$ registered phase is known to be unstable to the formation of topological defects and hence a fluid if $p<\sqrt{8}[15]$. As the hole stripes in the cuprates act as domain walls to the antiferromagnetic (AF) background, the SP is topologically equivalent to an IC phase near $p=2$ which led Zaanen et al. 12 to the conclusion that the SP in the cuprates is a stripe liquid. However, the instability of the membrane to both disorder pinning and formation of topological defects is rooted in the form of the entropic repulsion of classical strings. At low temperatures the fluctuations of the strings are no longer thermal but predominantly quantum. In this case the effective steric interaction between neighboring stripes decays exponentially with the distance rather than algebraically as in the purely thermal case [12]. In this work we study the influence of disorder and lattice perturbations in the quantum regime of the SP. We focus at the intermediate time- and length scale regime, as e.g. probed in neutron scattering experiments, where the dynamics is dominated by a single stripe rather than the collective and coherent membrane physics. The neighboring stripes nonetheless play an important role as they confine the stripe wandering. The stripe therefore cannot find the optimal path through the disorder potential.

We start with a phenomenological model of a stripe on a lattice in a disorder potential. Although our model describes stripes oriented along one of the simple lattice directions, the continuum limit we use below is not sensitive to the microscopic details, so that our results also apply for a SP with a diagonal orientation. Implicitly we assume that a particular SP order is well separated in energy from other configurations, so that through the RG the topology of the charge and spin order does not change. To study the dynamics of the stripe, it is then possible to use a first quantized formulation of the problem. Consider a directed string of holes (enumerated by $n$ ) on a square lattice with lattice constant $a$. Each hole is only allowed to hop in the transversal direction. To account for the stripe stiffness, we include a parabolic potential of strength $K$ which couples adjacent holes in the stripe. The Hamiltonian is then $H=H_{S}+H_{D}$,

$$
\begin{aligned}
& H_{S}=\sum_{n}\left[-2 t \cos \left(\frac{\hat{p}_{n} a}{\hbar}\right)+\frac{K}{2 a^{2}}\left(\hat{u}_{n}-\hat{u}_{n+1}\right)^{2}\right], \\
& H_{D}=\sum_{n} V_{n}\left(\hat{u}_{n}\right) .
\end{aligned}
$$

Here, $t$ is the hopping parameter and $\hat{p}_{n}, \hat{u}_{n}$ are canonical conjugate transversal momentum and position vari- 
ables of the $n$-th hole, respectively. The eigenvalues of $\hat{p}_{n}$ are restricted to $-\pi<p_{n} a / \hbar<\pi$. The eigenvalues of $\hat{u}_{n}$ are thus integer multiples of $a$. A model similar to $H_{S}$, without disorder but with additional curvature terms, was investigated in [16]. $H_{S}$ can be brought via the canonical transformation $\hat{\varphi}_{n}=\sum_{m<n} \hat{p}_{m}, \hat{\pi}_{n}=$ $\hat{u}_{n-1}-\hat{u}_{n}$ into a form previously studied within the context of 1D Josephson Junction arrays 17 and exhibits a $T=0$ Kosterlitz-Thouless (KT) 18] transition at a critical value of $t / K . H_{D}$ describes the interaction of the stripe with an uncorrelated disorder potential, $\left\langle V_{n}(u) V_{n^{\prime}}\left(u^{\prime}\right)\right\rangle_{D}=D \delta\left(u-u^{\prime}\right) \delta_{n, n^{\prime}}$, where $\langle\ldots\rangle_{D}$ denotes the gaussian average over the disorder ensemble and $D=\hbar^{2} c / \tau_{I}$. Here, $c$ is the characteristic velocity of the stripe excitations and $\tau_{I}$ is the impurity scattering time. Due to the stripe repulsion $|u|<L$, where $L$ is the average inter-stripe distance.

The partition function $Z=\operatorname{tr} \exp (-\beta H)$ can be transformed in the usual way into a path integral form. We introduce $\epsilon=\beta \hbar / M$ and insert $M-1$ times the resolution of unity into the trace. Using the Villain approximation $\exp (\ell \cos \phi) \simeq \sum_{q} \exp \left(-q^{2} /(2 \ell)+i q \phi\right)$ and integrating over the $p$ 's and $q$ 's we arrive at

$$
\begin{aligned}
Z \simeq & \sum_{\left\{u_{n m}\right\}} \exp \left\{-\frac{1}{\hbar} \sum_{m=0}^{M-1} \sum_{n}\left[\frac{\hbar^{2}}{4 t \epsilon a^{2}}\left(u_{n m+1}-u_{n m}\right)^{2}\right.\right. \\
& \left.\left.+\frac{\epsilon K}{2 a^{2}}\left(u_{n m}-u_{n+1, m}\right)^{2}+\epsilon V\left(u_{n m}\right)\right]\right\},
\end{aligned}
$$

where $m$ and $n$ are discrete indices for the imaginary time and longitudinal direction, respectively. After introducing $i=1, \ldots, N$ replicas and taking the continuum limit $\epsilon, a \rightarrow 0$, we find the replica action

$$
\begin{aligned}
S^{r}= & \sum_{i} S_{0}\left[\phi^{i}\right]+\frac{g}{a} \sum_{i} \int_{0}^{\hbar \beta} d \tau \int d y \cos \left(2 \sqrt{\pi} \phi^{i}\right) \\
& +\frac{D}{2 a L \hbar} \sum_{i, i^{\prime}} \int d y \int_{0}^{\hbar \beta} d \tau d \tau^{\prime} \\
& \cos \left[2 \sqrt{\pi} \delta\left(\phi^{i}(y, \tau)-\phi^{i^{\prime}}\left(y, \tau^{\prime}\right)\right)\right]
\end{aligned}
$$

where $\delta=a /(2 L)$ is essentially the stripe density. The gaussian action $S_{0}$ is given by

$$
S_{0}[\phi(y, \tau)]=\frac{\hbar}{2 \pi \mu} \int_{0}^{\hbar \beta} d \tau \int d y\left[\frac{1}{c}\left(\partial_{\tau} \phi\right)^{2}+c\left(\partial_{y} \phi\right)^{2}\right] .
$$

Here, we introduced the dimensionless fields $\phi^{i}=$ $\sqrt{\pi} u^{i} / a$. The dynamics of the free stripe is completely characterized by the velocity $c=a \sqrt{2 t K} / \hbar$ and the dimensionless parameter $\mu=\sqrt{2 t / K}$, which is a measure for the competition between kinetic and confining energies. The parameter $g$ is introduced to account for the lattice effects. In the derivation we have used $\delta(u)=(1 / 2 L) \sum_{l} e^{i \pi l u / L}$ and kept only the most relevant modes with $l= \pm 1$. The form of the delta function reflects the confinement of the stripe by its neighbors.

We now state more precisely under which conditions the interaction between neighboring stripes can be neglected. It is reasonable to assume that the stripe-stripe interaction is dominated by contact interaction resulting from a short range repulsion. A simple estimate for the range of validity of the single stripe description was proposed in [12]: equating the mean square transversal wandering, $\Delta u(y, \tau) \simeq \sqrt{\mu} a \pi^{-1} \ln \left[\left(y^{2}+c^{2} \tau^{2}\right) / a^{2}\right]$, with the average distance between stripes, $L$, immediately yields $y_{c}=\tau_{c} c \simeq a \exp [\pi /(4 \delta \sqrt{\mu})]$. Therefore, on time and length scales smaller than $y_{c}, \tau_{c}$, the physics is dominated by single stripe dynamics while for larger scales the interaction must be taken into account. Although we cannot rule out the importance of other possible sources of direct stripe-stripe interactions, as e.g. (screened) Coulomb forces or Casimir-type interaction [19], at least for large $L$ we expect them to be weak compared to the steric repulsion. However, should other forces dominate, the crossover would occur at scales smaller than $y_{c}, \tau_{c}$.

To obtain the phase diagram of the stripe in presence of both disorder and lattice potentials, we derive the RG differential equations by calculating the correlator $\left\langle\exp i \sqrt{\pi}\left[\phi^{i_{1}}\left(y_{1}, \tau_{1}\right)-\phi^{i_{2}}\left(y_{2}, \tau_{2}\right)\right]\right\rangle$ to lowest nonvanishing order in $g$ and $D$. Using the method developed by Giamarchi and Schulz [20], we find under the rescaling $a^{\prime}=e^{\ell} a$ the flow equations

$$
\begin{array}{rlrl}
\frac{d}{d \ell} \mathcal{D} & =\left(3-2 \pi \mu \delta^{2}\right) \mathcal{D}, & \frac{d}{d \ell} \mathcal{G} & =(2-\pi \mu) \mathcal{G}, \\
\frac{d}{d \ell} \mu & =-\frac{1}{2} \mu^{2}\left(\mathcal{G}^{2}+\mathcal{D}\right), \quad \frac{d}{d \ell} c=-\frac{1}{2} \mathcal{D} \mu c .
\end{array}
$$

where $\mathcal{D}=4 \pi^{2} D \delta^{2} a^{2} /\left(\hbar^{2} c^{2} L\right)$ and $\mathcal{G}=\pi^{3 / 2} g a /(c \hbar)$. Our present analysis is strictly correct only for time- and length scales smaller than $\tau_{c}, y_{c}$, at which the interaction between stripes becomes important. Nonetheless, these RG equations do strongly influence also the zero frequency and wave vector behavior of the stripe array as we will discuss below. We begin however the analysis ignoring these cutoffs.

With no disorder $(\mathcal{D}=0)$, the set of equations (2) reduces to the conventional KT form describing the roughening transition of the stripe at $\mu_{c 1}=2 / \pi$. For $\mu<\mu_{c 1}$, $\mathcal{G}$ diverges, signaling a pinning of the stripe by the underlying lattice. The stripe is flat on length scales larger than the lattice pinning length $L_{p 1} \propto \exp \left[A / \sqrt{\mu_{c 1}-\mu}\right]$ ( $A$ is a constant), has infinite stiffness and its excitations are massive. For $\mu>\mu_{c 1}$, the lattice potential is irrelevant and the system flows towards a gaussian fixed point with renormalized $\mu^{*}$, massless excitations, logarithmic wandering and strong quantum fluctuations.

In the presence of disorder there is another instability at $\mu_{c 2}=3 /\left(2 \pi \delta^{2}\right)$ at which $\mathcal{D}$ becomes relevant. 
If $\mu<\mu_{c 2}$ (and $\mathcal{G}=0$ ), $\mathcal{D}$ flows to infinity and the stripe is in a disordered (pinned) state. Near the transition it is possible to define a localization or collective pinning length $L_{p 2}$ of the stripe. Its asymptotic dependence on the disorder strength near the critical region (and $\mathcal{G}=0$ ) can be found from linearizing and integrating the RG-equations close to the singularity, $L_{p 2} \propto \exp \left[B \delta / \sqrt{\left(\mathcal{D}_{0}-\mathcal{D}_{c}\right)}\right]\left(B\right.$ is a constant), where $\mathcal{D}_{0}$ is the bare and $\mathcal{D}_{c}$ the critical disorder strength. Although the transverse excitations of the disorder pinned stripe are localized, the disorder does not lead to a gap in the density of states [21]. Further, contrary to the lattice pinned stripe, the stiffness of the disorder pinned stripe is finite: adding a small linear tilt to the fields $\phi^{i} \rightarrow \phi^{i}+\delta \phi y$ leaves the disorder term in the action (11) unchanged and the gaussian term in (1) is modified only at order $(\delta \phi)^{2}$. Hence, the kink free energy vanishes [21].

The diagram in Fig. 1 shows the stability regions of the stripe in the $\delta$ - $\mu$ plane, for $\mathcal{D}, \mathcal{G} \simeq 0$. If neither disorder nor lattice potentials are relevant, the stripe is in the freely fluctuating gaussian phase. On large scales, an array of coupled free gaussian strings can be described in terms of a $2+1 \mathrm{D}$ quantum membrane (QM). If the lattice is relevant and disorder is not, the stripe is in the flat phase. The disordered stripe, which exists if disorder is relevant, can be further distinguished whether or not the lattice pinning is a relevant perturbation. Even though disorder will always win in the long wavelength limit, the stripe will be locally flat if the lattice potential is relevant ("dis. flat") and if $L_{p 1}<L_{p 2}$. In the dis. flat phase the sharp band edge of the massive excitations which exist in the flat phase are washed out by the disorder and Lifshitz-like tails extend down to zero energy. No true gap survives but the lattice pinning reduces the low energy spectral weight and gives rise to a pseudogap. At $L_{p 1} \approx L_{p 2}$ the pseudogap disappears and a crossover to the disordered phase takes place.

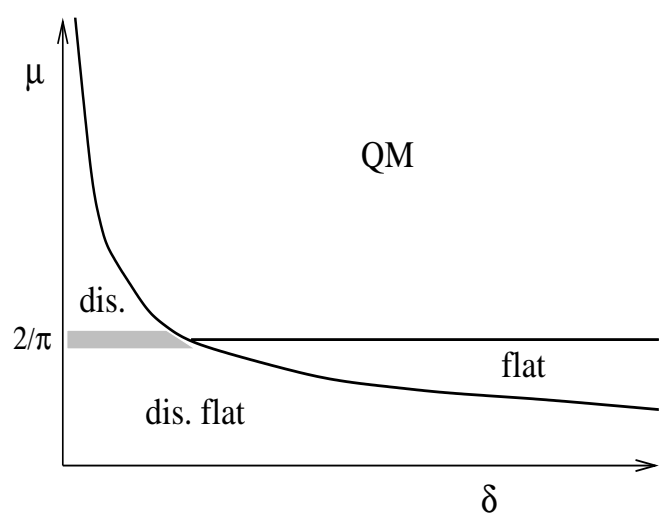

FIG. 1. Phase diagram of the stripe as a function of $\delta=a /(2 L)$ and $\mu=\sqrt{2 t / K}$.

¿From our analysis follows, that dynamical fluctuating SP order occurs only in a situation in which both disorder and lattice potentials are irrelevant, implying that the dynamical $\mathrm{SP}$ of the $\mathrm{La}_{2-\mathrm{x}} \mathrm{Sr}_{\mathrm{x}} \mathrm{CuO}_{4}$ compound must be insensitive to disorder. Indeed, the IC spin fluctuations [3] in this compound near optimal doping are strikingly similar to those found in $\mathrm{La}_{2} \mathrm{CuO}_{4+\delta}$ [5]. In $\mathrm{La}_{2} \mathrm{CuO}_{4+\delta}$ the interstitial oxygens are mobile down to temperature of about $200 \mathrm{~K}$ and produce annealed as opposed to the quenched disorder in the Sr doped compound. Although this shows that weak disorder is unimportant near optimal doping, at lower doping, and hence lower $\delta$ (for $\mathrm{La}_{2-\mathrm{x}} \mathrm{Sr}_{\mathrm{x}} \mathrm{CuO}_{4}, \delta \propto x$ 顿), we expect from Fig. 1 a critical doping $x_{c}$ at which the disorder becomes relevant. Hence, below $x_{c}$ the stripes are pinned, implying a broadening of the IC spin fluctuations. Eventually, the IC peaks will overlap to produce a single broad peak centered at the commensurate $\mathrm{AF}$ position, as has been observed in the spin glass phase $(x<0.05)$ in neutron scattering 俩. It is also likely that a new commensurate peak appears due to the growth of strong AF fluctuations and the appearance of phase-domain walls close to the AF ordered phase [22]. For disorder pinned stripes, a depinning transition under strong external fields has been predicted 23].

In oxygen doped nickelates [9, 10], the IC peak widths are always much narrower than in the $\mathrm{Sr}$ doped ones [8] so we conclude that disorder is relevant and hence $\mu<\mu_{c 2}$ for the nickelates. Further, both $\mathrm{Sr}$ and $\mathrm{O}$ doped nickelates show strong commensuration effects, i.e. the stripe spacing tends to lock in at values commensurate with the lattice spacing [8 10]. The width of a commensurate plateau depends on the competition between the effective stripe interaction and the strength of the lattice potential. To compare these two effects, one must integrate over the independent stripe fluctuations at smaller scales up to $y_{c}$. If $\mu<\mu_{c 1}$, the effective lattice coupling is strong and very wide commensurate plateaus as a function of either doping or temperature are expected. This is the situation in the nickelates. Similar commensuration effects are also observable in the cuprates, however, because $\mu>\mu_{c 1}$, the renormalized lattice coupling at $y_{c}$ is exponentially suppressed and only very narrow commensurate plateau exist. The SP is then nearly always in a floating phase IC with the lattice. This is consistent with the very weak Bragg peaks which are observable in $\mathrm{La}_{2-\mathrm{x}} \mathrm{Sr}_{\mathrm{x}} \mathrm{CuO}_{4}$ only at $x=1 / 8$ 24] and the static SP of $\mathrm{La}_{1.6-\mathrm{x}} \mathrm{Nd}_{0.4} \mathrm{Sr}_{\mathrm{x}} \mathrm{CuO}_{4}$ which is most pronounced again at $x \simeq 1 / 8$ [1] whereas at other compositions the IC Bragg peaks are much weaker and broader [2].

We now briefly discuss how the stripe fluctuations affect the correlations of the spin system, where we now consider an array of stripes with a harmonic coupling of strength $U$ between neighboring stripes. We first write the staggered spin density as $\mathbf{M}(\mathbf{r}, \tau)=$ $\mathbf{M}_{A F}(\mathbf{r}, \tau) M_{S}(\mathbf{r}, \tau)$ where $\mathbf{M}_{A F}$ describes the staggered spin density of the confined undoped regions and $M_{S}$ is a function which changes sign at the position of the domain walls. It can be shown that the inelastic part of the 
$\left\langle M_{S} M_{S}\right\rangle$ correlator can be written at $T=0$ as 25]

$$
\begin{gathered}
\left\langle M_{S} M_{S}\right\rangle_{\text {inel }}(\mathbf{k}, \omega) \propto \Gamma\left(k_{x} \pm \pi / L, k_{y}, \omega\right) e^{-k_{x}^{2}\left[\xi^{2}+a^{2} \Gamma_{0} / \pi\right]}, \\
\Gamma(\mathbf{k}, \omega) \propto\left[\omega^{2}+\omega_{k_{y}}^{2}+2 U c^{2} \mu\left(1-\cos k_{x} L\right) / a^{2}\right]^{-1}
\end{gathered}
$$

where $k_{x}\left(k_{y}\right)$ is the wave vector perpendicular (parallel) to the stripe orientation, $\xi$ the width of the stripes, $\Gamma_{0} \simeq \pi \mu^{3 / 4} /\left(4 U^{1 / 4}\right)$ the quantum analog of a DebyeWaller factor, $\Gamma$ the propagator of the stripe array and $\omega_{k_{y}}$ the single stripe dispersion. From the form of the Debye Waller factor it is seen that weak coupling (small $U$ ) and strong quantum fluctuations (large $\mu$ ) suppress the inelastic signal. Assuming, that the dynamics of $\left\langle M_{S} M_{S}\right\rangle$ and $\left\langle\mathbf{M}_{A F} \mathbf{M}_{A F}\right\rangle$ decouple, the $\langle\mathbf{M M}\rangle$ correlator becomes a simple convolution of $\left\langle\mathbf{M}_{A F} \mathbf{M}_{A F}\right\rangle$ and $\left\langle M_{S} M_{S}\right\rangle$ in $(\mathbf{k}, \omega)$-space. For commensurately pinned stripes the stripe fluctuations are suppressed, but long ranged AF order can give rise to dispersion at low energies through spin waves and an energy evolution as drawn qualitatively in Fig. 2a. Such an energy dependence of the peak width has been measured in $\mathrm{La}_{2} \mathrm{NiO}_{4.133}$ [10]. If both lattice and disorder are irrelevant, $\omega_{k_{y}}=c^{*} k_{y}$ and the stripe propagator supports both acoustic modes near $\mathbf{k}=0$ and optical modes at $k_{x}= \pm \pi / L$. In the cuprates, the AF order is short ranged which leads to a finite energy broad peak around $\mathbf{k}=( \pm \pi / a, \pm \pi / a)$ of $\left\langle\mathbf{M}_{A F} \mathbf{M}_{A F}\right\rangle$. The evolution of the IC signal has qualitatively the form as shown in Fig. $2 \mathrm{~b}$, with a dispersion (due to stripe excitations) away from the IC position to the commensurate position at larger energies. The dispersion is not symmetric around the IC positions because the Debye Waller factor suppresses signals far from the AF wave vector. This simple picture actually describes the experimentally observed evolution of the IC peaks in $\mathrm{La}_{2-x} \mathrm{Sr}_{\mathrm{x}} \mathrm{CuO}_{4}$ [26]. In the disorder dominated SP, localized single stripe excitations lead to a broad spectral weight distribution in $\mathbf{k}$ space at low energies (Fig. 2 c) resulting in a broad featureless peak centered at $(\pi / a, \pi / a)$, as is observed in the spin glass phase of the cuprates [4].

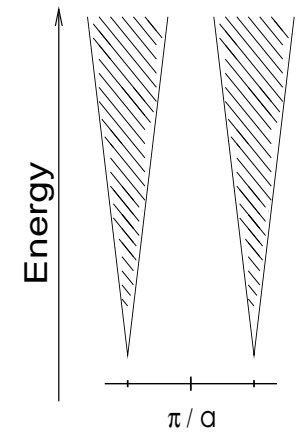

a)

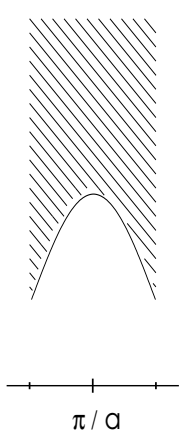

b)

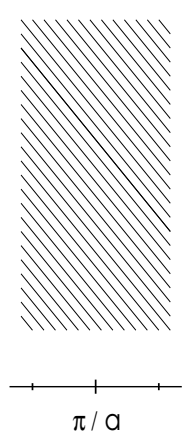

c)
FIG. 2. Evolution of the IC signal with energy: a) for commensurate lattice pinned stripes and long range AF order; b) in the QM phase; c) for a strongly disordered SP.
In conclusion, using a perturbative RG calculation we studied the competition between disorder and lattice effects on an effective model for transverse stripe fluctuations. We identified three different phases, depending on the stripe density $\delta$, the ratio between the kinetic to the confining energy of the stripes $(\mu)$, the strength of disorder $(\mathcal{D})$ and the lattice strength $(\mathcal{G})$ : pinned by disorder, pinned by the lattice and the QM phase. Given the experimental data in cuprates and nickelates we can locate these materials in our phase diagram and predict crossovers and phase transitions between the distinct phases. Using a simple model calculation, we discussed the energy evolution of the IC spin fluctuations and obtained qualitative agreement with the experiments.

We thank A. O. Caldeira, G. Castilla, M. P. A. Fisher, A. van Otterlo and H. Schmidt for helpful discussions. N. H. acknowledges support from the Gottlieb Daimlerund Karl Benz-Stiftung and the Graduiertenkolleg "Physik nanostrukturierter Festkörper", Univ. Hamburg. A. H. C. N. acknowledges support from the Alfred P. Sloan foundation and the US Department of Energy.

[1] J. M. Tranquada et al., Nature 365, 561 (1995); J. M. Tranquada et al., Phys. Rev. B 54, 7489 (1996).

[2] J. M. Tranquada et. al., Phys. Rev. Lett. 78, 338 (1997).

[3] G. Aeppli et al., Science 278, 1432 (1997); T. E. Mason et al., Phys. Rev. Lett. 68, 1414 (1992).

[4] K. Yamada et al., Phys. Rev. B 57, 6165 (1998).

[5] B. O. Wells et al., Science 277, 1067 (1997).

[6] P. Dai et al., Phys. Rev. Lett. 80, 1738 (1998).

[7] N. L. Saini et al., Phys. Rev. Lett. 79, 3467 (1997).

[8] G. Blumberg et al., Phys. Rev. Lett. 80, 564, (1998); S. H. Lee and S.-W. Cheong, ibid. 79, 2514, (1997); J. M. Tranquada et al., Phys. Rev. B 54, 12318 (1996).

[9] P. Wochner et al., Phys. Rev. B 57, 1066 (1998); K. Nakajima et al., J. Phys. Soc. Jpn. 66, 809 (1997); J. M. Tranquada et al., Phys. Rev. Lett. 73, 1003 (1994).

[10] J. M. Tranquada et al., Phys. Rev. Lett. 79, 2133 (1997).

[11] A. H. Castro Neto and F. Guinea, Phys. Rev. Lett. 80, 4040 (1998); M. I. Salkola et al., ibid. 77, 155 (1996).

[12] J. Zaanen et al., Phys. Rev. B 53, 8671 (1996).

[13] A. H. Castro Neto and D. Hone, Phys. Rev. Lett. 76, 2165 (1996).

[14] T. Nattermann et al., Europhys. Lett. 16, 295 (1991). However, in the presence of soft wall-wall repulsion, a depinning transition can occur, see e.g. T. P. Devereaux et al., Phys. Rev. B 50, 13625 (1994).

[15] S. N. Coppersmith et al., Phys. Rev. B 25, 349 (1982).

[16] H. Eskes et al., Phys. Rev. B 58, 6963 (1998).

[17] R. M. Bradley and S. Doniach, Phys. Rev. B 30, 1138 (1984).

[18] J. M. Kosterlitz and D. J. Thouless, J. Phys. C 6, 118 (1973).

[19] L. P. Pryadko et al., Phys. Rev. Lett. 80, 5651 (1998). 
[20] T. Giamarchi and H. J. Schulz, Phys. Rev. B 37, 325 (1988).

[21] M. P. A. Fisher et al., Phys. Rev. B 40, 546 (1989).

[22] A. H. Castro Neto, Zeitsch. Phys. B 103, 185 (1997).

[23] C. Morais Smith et al., Phys. Rev. B 58, 453 (1998).

[24] T. Suzuki et al., Phys. Rev. B 57, R3229 (1998).

[25] N. Hasselmann et al.; unpublished.

[26] S. M. Hayden et al., Phys. Rev. Lett. 76, 1344 (1996); T. E. Mason et al., ibid. 77, 1604 (1996). 